\title{
ON EVALUATION \\ OF HIGHER ORDER COHOMOLOGY OPERATIONS
}

\section{LEIF KRISTENSEN and IB MADSEN}

\section{Introduction.}

It is the purpose of the present paper to treat the problem of evaluation of secondary and tertiary operations in low dimensions, generalizing the statement

$$
\begin{array}{lll}
S q^{n+1}(\hat{x})=0 & \text { if } & \operatorname{deg} \hat{x} \leqq n, \\
S q^{n+1}(\hat{x})=\hat{x}^{2} & \text { if } & \operatorname{deg} \hat{x}=n+1 .
\end{array}
$$

In the case of secondary operations, this was done in [4]; however, the treatment given here is very simple and easy. The result in the case of tertiary operations is new. The proof in this case is somewhat involved. We hope to be able to simplify the proof and to generalize the result further. Some applications of the results will be given in a forthcoming paper. Applications of the result for secondary operations have been given by Mahowald [6]. A general definition of operations of the $N$-th kind has been given by Maunder in [7].

Let $A$ denote the Steenrod algebra $(\bmod 2)$. Let

$$
A(m)=A / B(m),
$$

where $B(m)$ is the left ideal of elements of excess $\geqq m+1$. To each relation in the left $A$-module $A(m)$,

$$
r: \quad 0=\sum \hat{\alpha}_{v} \hat{a}_{v},
$$

there is associated a secondary operation $Q u^{r}$, defined in degrees less than $m+1$. The operation $Q u^{r}$ is determined up to a primary operation. Let us consider the relation

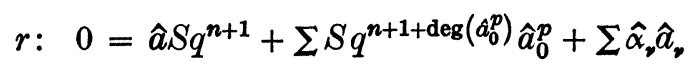

in $A(m), m>n$, where excess $\left(\hat{\alpha}_{v} \hat{a}_{v}\right)>n+1$ and $\sum \hat{a}_{0}^{p}$ appears as middle term in the Cartan formula for $\hat{a}$ :

$$
\Delta(\hat{a})=\Sigma \hat{a}^{\prime} \otimes \hat{a}^{\prime \prime}+\Sigma \hat{a}_{0}^{p} \otimes \hat{a}_{0}^{p}+\Sigma \hat{a}^{\prime \prime} \otimes \hat{a}^{\prime} .
$$

Received August 10, 1966. 
Then we have

Theorem. There exists an operation $Q u^{r}$ associated with $r$ taking the values

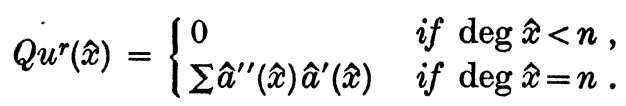

Applied to the stable operations $\Phi_{i, j}, i \leqq j$ and $i \neq j-1$, introduced by Adams [1], it yields

$$
\Phi_{i, j}(\hat{x})=0 \text { if } \operatorname{deg} \hat{x} \leqq \begin{cases}2^{i}-1 & \text { if } i=j, \\ 2^{j}-2^{i}-1 & \text { if } i<j-1 .\end{cases}
$$

It is well known that a tertiary operation is associated with a relation between primary and secondary operations:

$$
\sum \hat{a}_{i} Q u^{r_{i}}+Q u^{\varepsilon}=0,
$$

where $\hat{a}_{i} \in A, r_{i}$ and $\varepsilon$ are relations in $A$. The tertiary operation is defined in dimensions less than the excess of $\varepsilon$ minus 1 (for definition see Section 2). It is defined at least on classes $\hat{x}$ annihilated by all primary operations $\hat{\alpha} \in A$ and by the secondary operations $Q u^{r_{i}}$.

Let us consider the relation

$$
\mathscr{R}: \sum \hat{a}_{i} Q u^{r_{i}}+S q^{n+1+\operatorname{deg} r_{0}} Q u^{r_{0}}+Q u^{\varepsilon},
$$

where $\varepsilon$ has excess $\geqq n+2$ and $r_{i}$ is the relation

$$
\begin{array}{ll}
r_{i}: & 0=\hat{b}_{i} S q^{n+1}+\sum S q^{n+1+\operatorname{deg} \hat{b}_{i, 0}^{q}} \hat{b}_{i, 0}^{q}+(\text { terms of excess } \geqq n+2), \\
r_{0}: & 0=\sum \hat{a}_{i, 0}^{p} \hat{b}_{i, 0}^{q} .
\end{array}
$$

Here $\hat{a}_{i, 0}^{p}$ (resp. $\hat{b}_{i, 0}^{q}$ ) appears as middle term in the Cartan formula for $\hat{a}_{i}$ (resp. $\hat{b}_{i}$ ), that is,

$$
\begin{aligned}
& \Delta\left(\hat{a}_{i}\right)=\sum \hat{a}_{i, s}^{\prime} \otimes \hat{a}_{i, s}^{\prime \prime}+\sum \hat{a}_{i, 0}^{p} \otimes \hat{a}_{i, 0}^{p}+\sum \hat{a}_{i, 8}^{\prime \prime} \otimes \hat{a}_{i, s}^{\prime}, \\
& \Delta\left(\hat{b}_{i}\right)=\sum \hat{b}_{i, t}^{\prime} \otimes \hat{b}_{i, t}^{\prime \prime}+\sum \hat{b}_{i, 0}^{p} \otimes \hat{b}_{i, 0}^{p}+\sum \hat{b}_{i, t}^{\prime \prime} \otimes \hat{b}_{i, t}^{\prime},
\end{aligned}
$$

where $\operatorname{deg} \hat{a}_{i, \ell}^{\prime}<\operatorname{deg} \hat{a}_{i, 8}^{\prime \prime}$ and $\operatorname{deg} \hat{b}_{i, t}^{\prime}<\operatorname{deg} \hat{b}_{i, t}^{\prime \prime}$. Then we have

THEOREM. There exists a tertiary operation $Q u^{\mathscr{R}}$ associated with $\mathscr{R}$ taking the values

$$
Q u^{\mathscr{R}}(\hat{x})= \begin{cases}0 & \operatorname{deg} \hat{x} \leqq n-1, \\ Q u^{r}(\hat{x}) \hat{x} & \operatorname{deg} \hat{x}=n\end{cases}
$$

where $Q u^{r}$ is the secondary operation associated with the relation $r: 0=$ $\Sigma \hat{a}_{i} \hat{b}_{i}$. 
A more detailed statement of this theorem is given in Section 4, Theorem 2.

In Section 2 we review the definitions of secondary and tertiary operations in terms of cochain operations. In this connection we define a certain "Massey product" in the Steenrod algebra.

Section 3 contains cochain formulas used in the proof of the theorems mentioned above. Detailed statements of the theorems and proofs are given in Section 4.

The method of proof is based on exact sequences of the form

$$
\left.\mathcal{O}^{m} \stackrel{\nabla}{\longrightarrow} Z^{m} \stackrel{\bullet}{\longrightarrow} A \oplus \ldots \oplus A \rightarrow 0 \quad \text { ( } m \text { summands }\right)
$$

$$
\mathcal{O}_{(2)} \stackrel{\nabla}{\longrightarrow} Z \mathcal{O}_{(2)} \stackrel{\bullet}{\longrightarrow} A \otimes A \rightarrow 0 \text {. }
$$

For definitions and proofs see [4] or [3] in case (1), and [5] or [3] in case $(2)$. (In $[5], \mathcal{O}_{(2)}$ is denoted by $Q$.)

\section{Definitions.}

Let $F$ denote the free associative $Z_{2}$-algebra with unit generated by $s q^{i}, i=1,2, \ldots$ Let $R$ be the subalgebra generated by the Adem relations. Then $F / R=A$, such that we have an exact sequence

$$
0 \rightarrow R \rightarrow F \rightarrow A \rightarrow 0 .
$$

Elements of $F$ will be denoted by small letters $\alpha, \beta, a, b, \ldots$. The corresponding elements in $A$ will be denoted by $\hat{\alpha}, \hat{\beta}, \hat{a}, \hat{b}, \ldots$. Recall that the excess of a monomial $s q^{i_{1}} s q^{i_{2}} \ldots s q^{i_{r}}$ in $F$ is

$$
\max _{j}\left(i_{j}-i_{j+1}-\ldots-i_{r}\right) \text {, }
$$

and that the excess of a sum $\Sigma m_{i}$ of monomials is $\min _{i} \operatorname{exc}\left(m_{i}\right)$.

Also, to each element $\alpha$ in $F$ we associate a primary cochain operation $\alpha \in Z O^{1}$, such that if $\alpha$ has excess $n$, then $\alpha(u)=0$ if $u$ is a cochain of degree less than $n-1$ or a cocycle of degree $n-1$ (see p. 58 in [4]). We shall often identify $F$ with its image in $Z \mathcal{O}^{1}$.

Let $\left(b_{i j}\right)$ be a $n \times n$-matrix in elements of $F$ and let $a_{i}, c_{i}, e_{i}$ and $f_{i}$, $i=1,2, \ldots, n$, be elements from $F$ such that

$$
r_{i}=\Sigma b_{i j} c_{j}+f_{i} \text { and } s_{j}=\Sigma a_{i} b_{i j}+e_{j}
$$

map to zero in $A$, that is, $r_{i}$ and $s_{j}$ are relations in $A$. We obtain an identity in $F$ :

$$
\mathscr{R}: \sum a_{i} r_{i}+\sum s_{j} c_{j}+\varepsilon=0,
$$

where

$$
\varepsilon=\Sigma a_{i} f_{i}+\sum e_{j} c_{j}
$$


is a relation. We shall refer to (3) as a relation among relations with an unfactorized relation $\varepsilon$.

Let us define $N$ to be the largest number such that the inequalities

are fulfilled.

$$
N \leqq \operatorname{exc} f_{i}-2, \quad N \leqq \operatorname{exc} e_{j}-\operatorname{exc} c_{j}-2
$$

If we consider the relations $r_{i}, s_{j}$ and $\varepsilon$ as cochain operations, then because of the exactness of the sequence

$$
\stackrel{\nabla}{\longrightarrow} Z \mathcal{O} \stackrel{\leftrightarrow}{\longrightarrow} A \rightarrow 0,
$$

where $\nabla \theta=\delta \theta+\theta \delta$, there exist operations $R_{i}, S_{j}$ and $E$ such that

$$
\nabla R_{i}=r_{i}, \quad \nabla S_{j}=s_{j}, \quad \nabla E=\varepsilon .
$$

Moreover, $E$ can and shall be chosen such that $E(u)=0$ if $u$ is a cochain of degree less than $N$ or an $N$-dimensional cocycle [4, Theorem 3.7]. This is of importance in the definition of tertiary cohomology operations associated with $\mathscr{R}$ (see below).

We now proceed to the definitions of secondary and tertiary cohomology operations. First we describe what we shall understand by a secondary operation associated with the relation

$$
r_{i}=\Sigma b_{i j} c_{j}+f_{i} \text {. }
$$

Let $X$ be a CSS-complex and $\left(x,\left\{w_{j}\right\}\right)$ a system of cochains on $X$ with $\operatorname{deg} x \leqq \operatorname{exc} f_{i}-1$, satisfying

$$
\delta x=0, \quad \delta w_{j}=c_{j}(x) .
$$

Define a cocycle $q u^{r_{i}}\left(x,\left\{w_{j}\right\}\right)$ on $X$ by

$$
q u^{r_{i}}\left(x,\left\{w_{j}\right\}\right)=R_{i}(x)+\Sigma b_{i j}\left(w_{j}\right) .
$$

It is clear that $q u^{r_{i}}\left(x,\left\{w_{j}\right\}\right)$ depends on the choice of $R_{i}$. However, since two choices differ at most by a primary operation, it follows that $q u^{r_{i}}\left(x,\left\{w_{j}\right\}\right)$ is determined by $r_{i}$ modulo a cocycle $\alpha(x), \alpha \in Z \mathcal{O}$.

Define natural additive relations $Q u^{r_{i}}$ from $H^{*}(X)$ to $H^{*}(X)$ as follows:

The definition domain $\operatorname{Def}\left(Q u^{\tau_{i}}\right)$ is the set of classes in $H^{*}(X)$ of degree less than $\operatorname{exc} f_{i}$ and annihilated by $\hat{c}_{j}, j=1, \ldots, n$.

For $\hat{x}$ in $\operatorname{Def}\left(Q u^{r_{i}}\right)$ put $Q u^{r_{i}}(\hat{x})$ equal to the factor set of cohomology classes of $q u^{r_{i}}\left(x,\left\{w_{j}\right\}\right)$ where $\left(x,\left\{w_{j}\right\}\right)$ runs over all systems of cochains satisfying (5). The indeterminacy subgroup $\operatorname{Ind}\left(Q u^{r_{i}}\right)$ is the relevant component of the graded group $\sum \hat{b}_{i j} H^{*}(X)$. By an earlier remark it is 
clear that $Q u^{r_{i}}$, although not pointed out in the notation, is only determined up to a primary cohomology operation.

An easy computation (carried out in [4]) shows that $Q u^{r_{i}}$ satisfies the following additivity relations

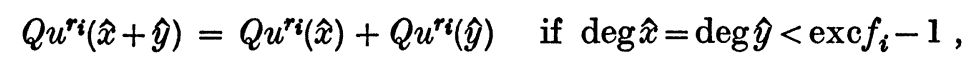

$$
Q u^{r_{i}}(\hat{x}+\hat{y})=Q u^{r_{i}}(\hat{x})+Q u^{r_{i}}(\hat{y})+d\left(f_{i} ; x, y\right)^{\wedge} \quad \text { if } \operatorname{deg} \hat{x}=\operatorname{deg} \hat{y}=\operatorname{exc} f_{i}-1 \text {. }
$$

Here $d\left(f_{i} ; x, y\right)$ is a cochain operation measuring the deviation from additivity of $f_{i}$, that is,

$$
\begin{gathered}
\delta d\left(f_{i} ; x, y\right)+d\left(f_{i} ; \delta x, \delta y\right)=f_{i}(x+y)+f_{i}(x)+f_{i}(y), \\
d\left(f_{i} ; x, 0\right)=0 \text { and } d\left(f_{i} ; 0, y\right)=0 .
\end{gathered}
$$

The existence of such an operation is a trivial consequence of the exact sequence

$$
\mathcal{O}^{2} \stackrel{\nabla}{\longrightarrow} Z \mathcal{O}^{2} \stackrel{\bullet}{\longrightarrow} A \oplus A \rightarrow 0
$$

mentioned in Section 1. However, in order to evaluate $d\left(f_{i} ; x, y\right)$ one has to give a more constructive definition. This is done in (6) and (7) of Section 3.

REMaRK. The identities (6) shall be understood with care, i.e. to each choice of the operations $Q u^{r_{i}}$ and $d\left(f_{i} ; x, y\right)$ the identities hold modulo the total indeterminacy involved:

If $f_{i}=s q^{n}$, then one can choose the operation $d\left(f_{i} ; x, y\right)$ such that for $\operatorname{deg} x=\operatorname{deg} y<\operatorname{exc} f_{i}-1$ :

$$
d\left(f_{i} ; x, y\right)=0
$$

for $\operatorname{deg} x=\operatorname{deg} y=\operatorname{exc} f_{i}-1$ :

$$
d\left(f_{i} ; x, y\right)=x y \text {. }
$$

This was proved already in [4]; (6) of Section 3 repeats this proof.

Next, let us turn to the case of tertiary operations. Define a cochain operation $M$ by

$$
\begin{aligned}
M(x)=\sum a_{i} R_{i}(x)+\sum S_{j} c_{j}(x) & +E(x)+\sum d\left(a_{i} ; \delta R_{i} x, R_{i} \delta x\right)+ \\
& +\sum d\left(a_{i} ; b_{i 1} c_{1}(x), \ldots, b_{i n} c_{n}(x), f_{i}(x)\right) .
\end{aligned}
$$

An easy check shows that $\nabla M=0$; that is, $M$ is a primary cochain operation.

The element of Steenrod's algebra associated with $M$ is called the Massey product of the relation among relations $\mathscr{R}$. The cochain opera- 
tions $R_{i}$ and $S_{j}$ are determined only modulo primary cochain operations (i.e. elements in $Z \mathcal{O}$ ). Furthermore, it was assumed that $E$ was chosen such that $E(x)=0$ whenever $x$ is a $N$-dimensional cocycle or a cochain of dimension less than $N$. Therefore, the Massey product of $\mathscr{R}$ has the indeterminacy

$$
\sum \hat{a}_{i} A+\sum A \hat{c}_{j}+B(N),
$$

where $B(N)$ is the left ideal in $A$ of elements of excess larger than $N$.

Now, assume that $\hat{M}=\sum \hat{\mu}_{k} \hat{m}_{k}$ is a factorization of $\hat{M}$ in $A$. Then $M+\sum \mu_{k} m_{k}$ is in $Z \mathcal{O}$ and maps to zero in $A$ (cf. the exact sequence (4)). Choose by exactness a cochain operation $\chi$ with

$$
\nabla \chi=M+\Sigma \mu_{k} m_{k} .
$$

Let us consider systems of cochains $\left(x,\left\{w_{j}\right\},\left\{u_{i}\right\},\left\{v_{k}\right\}\right)$, where

$$
\delta x=0, \quad \delta w_{j}=c_{j}(x), \quad \delta u_{i}=q u^{r_{i}}\left(x,\left\{w_{j}\right\}\right), \quad \delta v_{k}=m_{k}(x) .
$$

For each such system satisfying in addition $\operatorname{deg} x \leqq N$, the cochain $q u^{\mathscr{R}}\left(x,\left\{w_{j}\right\},\left\{u_{i}\right\},\left\{v_{k}\right\}\right)$ given by

$$
\text { (11) } \begin{aligned}
q u^{\mathscr{R}}\left(x,\left\{w_{j}\right\},\left\{u_{i}\right\},\left\{v_{k}\right\}\right)= & \chi(x)+\sum a_{i}\left(u_{i}\right)+\sum S_{j}\left(w_{j}\right)+\sum \mu_{k}\left(v_{k}\right)+ \\
& +\sum d\left(a_{i} ; b_{i 1}\left(w_{1}\right), \ldots, b_{i n}\left(w_{n}\right), q u^{r_{i}}\left(x,\left\{w_{j}\right\}\right)\right)
\end{aligned}
$$

is actually a cocycle. One may therefore define a tertiary cohomology operation corresponding to $\mathscr{R}$ in the following way:

The domain of definition $\operatorname{Def}\left(Q u^{\mathscr{R}}\right)$ consists of all elements in $H^{*}(X)$ of degree less than or equal to $N$ annihilated by the operations $\hat{c}_{j}, Q u^{r_{i}}$ and $\hat{m}_{k}$.

If $\hat{x} \in \operatorname{Def}\left(Q u^{\mathscr{R}}\right)$, then put $Q u^{\mathscr{R}}(\hat{x})$ equal to the factor set of cohomology classes of

$$
q u^{\mathscr{R}}\left(x,\left\{w_{j}\right\},\left\{u_{i}\right\},\left\{v_{k}\right\}\right),
$$

where $\left(x,\left\{w_{j}\right\},\left\{u_{i}\right\},\left\{v_{k}\right\}\right)$ runs over all systems of cochains satisfying (10).

The indeterminacy subgroup $\operatorname{Ind}\left(Q u^{\mathscr{R}}\right)$ is the appropriate component of the graded group

$$
\sum \hat{a}_{i} H^{*}(X)+\sum Q u^{s_{j}} H^{*}(X)+\sum \hat{\mu}_{k} H^{*}(X) .
$$

Although it has not been made clear in the notation, the definition of the cochain

$$
q u^{\mathscr{R}}\left(x,\left\{w_{j}\right\},\left\{u_{i}\right\},\left\{v_{k}\right\}\right)
$$

involves choices of the cochain operations $M$ and $\chi$. Hence the natural additive relation $Q u^{\mathscr{R}}$ is not uniquely determined by $\mathscr{R}$. Two choices of $Q u^{\mathscr{R}}$ differ at most by a secondary operation. 
As in the secondary case, one can prove by a lengthy but straightforward computation that

$$
\begin{array}{ll}
Q u^{\mathscr{R}}(\hat{x}+\hat{y})=Q u^{\mathscr{R}}(\hat{x})+Q u^{\mathscr{R}}(\hat{y}) & \text { if } \operatorname{deg} \hat{x}=\operatorname{deg} \hat{y}<N, \\
Q u^{\mathscr{R}}(\hat{x}+\hat{y})=Q u^{\mathscr{R}}(\hat{x})+Q u^{\mathscr{R}}(\hat{y})+d(E ; x, y)^{\wedge} & \text { if } \operatorname{deg} \hat{x}=\operatorname{deg} \hat{y}=N,
\end{array}
$$

where $d(E ; x, y)$ is a cochain operation of excess $N$, for which

$$
\delta d(E ; x, y)+d(E ; \delta x, \delta y)=E(x+y)+E(x)+E(y)+d(\varepsilon ; x, y),
$$

and $d(E ; x, y)=0$ if $x=0$ or $y=0$.

Again, (12) shall be understood in the following way: To each choice of the operations $Q u^{\mathscr{R}}$ and $d(E ; x, y)$, the identities are fulfilled modulo the total indeterminacy involved.

If $\varepsilon=\nabla E$ has the form

$$
\varepsilon=e s q^{N+2}+\sum s q^{N+2+\operatorname{deg} e_{0}^{p}} e_{0}^{p}+(\text { terms of higher excess }),
$$

then there is a choice of $d(E ; x, y)$ such that

for $\operatorname{deg} x=\operatorname{deg} y<N$ :

$$
d(E ; x, y)=0
$$

for $\operatorname{deg} x=\operatorname{deg} y=N$ and $\delta x=\delta y=0$ :

$$
d(E ; x, y)=\sum e_{i}^{\prime \prime}(x) \cdot e_{i}^{\prime}(y) \quad\left(\frac{1}{2} \text { Cartan formula }\right),
$$

where

$$
\Delta(\hat{e})=\sum \hat{e}_{i}^{\prime} \otimes \hat{e}_{i}^{\prime \prime}+\sum \hat{\epsilon}_{0}^{p} \otimes \hat{e}_{0}^{p}+\sum \hat{e}_{i}^{\prime \prime} \otimes \hat{e}_{i}^{\prime} \quad\left(\operatorname{deg} \hat{e}_{i}^{\prime}<\operatorname{deg} \hat{e}_{i}^{\prime \prime}\right)
$$

is the Cartan formula for $\hat{e}$. This is proved in Section 3.

Note that $Q u^{\mathscr{R}}$ is a stable operation if $\varepsilon=0$.

We close up this section by constructing universal examples for the stable tertiary operations. Consider the Postnikov system

$$
\begin{aligned}
& F_{2}(n) \rightarrow K_{(3)}(n) \\
& \downarrow \pi_{3} \\
& F_{1}(n) \rightarrow K_{(2)}(n) \stackrel{\Pi Q u^{i} i \times \Pi \hat{m}_{k}}{\longrightarrow} B_{(2)}(n) \\
& \downarrow \pi_{2} \\
& K\left(Z_{2}, n\right) \stackrel{\Pi \hat{c}_{j}}{\longrightarrow} B_{(1)}(n),
\end{aligned}
$$

where 


$$
\begin{aligned}
& F_{(2)}(n)=\Pi K\left(Z_{2}, n+\operatorname{deg} Q u^{r_{i}}-1\right) \times \Pi K\left(Z_{2}, n+\operatorname{deg} \hat{m}_{k}-1\right), \\
& F_{(1)}(n)=\Pi K\left(Z_{2}, n+\operatorname{deg} \hat{c}_{j}-1\right), \\
& B_{(2)}(n)=\Pi\left(Z_{2}, n+\operatorname{deg} Q u^{r_{j}}\right) \times \Pi K\left(Z_{2}, n+\operatorname{deg} \hat{m}_{k}\right), \\
& B_{(1)}(n)=\Pi K\left(Z_{2}, n+\operatorname{deg} \hat{c}_{j}\right) .
\end{aligned}
$$

As indicated on the diagram, the fibration

$$
K_{(2)}(n) \stackrel{\pi_{2}}{\longrightarrow} K\left(Z_{2}, n\right)
$$

has $k$-invariant $\Pi \hat{c}_{j}$, and the fibration

$$
K_{(3)}(n) \stackrel{\pi_{3}}{\longrightarrow} K_{2}(n)
$$

has $k$-invariant $\Pi Q u^{r_{i}} \times \Pi \hat{m}_{k}$.

Let $\hat{z}^{e}$ denote the fundamental class in $H^{e}\left(K\left(Z_{2}, e\right) ; Z_{2}\right)$. Then the class

$$
\sum \hat{a}_{i}\left(\hat{z}^{n+\operatorname{deg} Q u^{r_{i}-1}}\right)+\sum \hat{\mu}_{k}\left(\hat{z}^{n+\operatorname{deg} \hat{m}_{k-1}}\right)
$$

in the fiber $F_{(2)}(n)$ of the fibration

$$
F_{(2)}(n) \rightarrow K_{(3)}(n) \stackrel{\pi_{3}}{\longrightarrow} K_{(2)}(n)
$$

is transgressiv and transgresses into

$$
\sum \hat{a}_{i} Q u^{r_{i}} \pi_{2}{ }^{*}\left(\hat{z}^{n}\right)+\sum \hat{\mu}_{k} \hat{m}_{k} \pi_{2}^{*}\left(\hat{z}^{n}\right)=0 .
$$

If $\hat{u} \in H\left(K_{(3)}(n) ; Z_{2}\right)$ is a class which restricts to the above mentioned class in the fiber, then $\left(K_{(3)}(n), \hat{u}\right)$ is clearly a universal example for $Q u^{\mathfrak{R}}$.

\section{Some formulas.}

In this section we shall specialize the relations and the relation among relations in consideration in order to simplify the formulas.

Let $\hat{a} \in A$ and let

$$
\Delta(\hat{a})=\Sigma \hat{a}_{i}^{\prime} \otimes \hat{a}_{i}^{\prime \prime}+\sum \hat{a}_{0}^{p} \otimes \hat{a}_{0}^{p}+\sum \hat{a}_{i}^{\prime \prime} \otimes \hat{a}_{i}^{\prime} \quad\left(\operatorname{deg} \hat{a}_{i}^{\prime}<\operatorname{deg} \hat{a}_{i}^{\prime \prime}\right)
$$

be the Cartan formula for $\hat{a}$. Then $\Sigma \hat{a}_{0}^{p}$ has the property $\hat{a}\left(x^{2}\right)=\Sigma \hat{a}_{0}^{p}(x)^{2}$ for all cohomology classes $\hat{x}$. Hence there is a relation in $A$ :

$$
s: 0=\hat{a} S q^{n+1}+\sum S q^{n+1+\operatorname{deg} \hat{a}_{0}^{p}} \hat{a}_{0}^{p}+\hat{e},
$$

where $\hat{e}$ has excess larger than $n+1$. Let

$$
s=a s q^{n+1}+\sum s q^{n+1+\operatorname{deg} a_{0}^{p}} a_{0}^{p}+e
$$

denote the corresponding cochain operation (in $Z \mathcal{O}$ ). (Note, that this, in particular, means that $e(x)=0$ whenever $x$ is an $n$-dimensional cocycle or a cochain of dimension less than $n$.) As mentioned in Section 1 there is an exact sequence 


$$
\mathcal{O}_{(2)} \stackrel{\nabla}{\longrightarrow} Z \mathcal{O}_{(2)} \stackrel{\bullet}{\longrightarrow} A \otimes A \rightarrow 0,
$$

$\nabla$ being the differential

$$
(\nabla \theta)(x, y)=\delta \theta(x, y)+\theta(\delta x, y)+\theta(x, \delta y) .
$$

From (2) we conclude that there exists a cochain operation $T_{a} \in \mathcal{O}_{(2)}$ with the property

$$
\begin{aligned}
\delta T_{a}(x, y)+ & T_{a}(\delta x, y)+T_{a}(x, \delta y) \\
= & a(x y)+\sum a_{i}^{\prime}(x) a_{i}^{\prime \prime}(y)+\sum a_{0}^{p}(x) a_{0}^{p}(y)+\sum a_{i}^{\prime \prime}(x) a_{i}^{\prime}(y)+ \\
& +d(a ; \delta x y, x \delta y)+\operatorname{deg} x d(a ; x \delta y, x \delta y) .
\end{aligned}
$$

Using $T_{a}$ we can now (at least in low dimensions) give an explicit expression for a cochain operation $\theta(x)$ with

$$
\delta \theta(x)+\theta(\delta x)=s(x) .
$$

First, let $\theta^{\prime}(x)$ be the following partially defined cochain operation:

for $\operatorname{deg} x \leqq n-2$ :

$$
\theta^{\prime}(x)=0 \text {, }
$$

for $\operatorname{deg} x=n-1$ :

(4)

$$
\theta^{\prime}(x)=\Sigma a_{i}^{\prime \prime}(x) a_{i}^{\prime}(\delta x),
$$

for $\operatorname{deg} x=n$ :

$$
\theta^{\prime}(x)=\sum a_{i}^{\prime \prime}(x) a_{i}^{\prime}(x)+\sum a_{i}^{\prime}(x) \cup_{1} a_{i}^{\prime \prime}(\delta x)+T_{a}(x, \delta x),
$$

for $\operatorname{deg} x=n+1$ and $\delta x=0$ :

$$
\theta^{\prime}(x)=\Sigma a_{i}^{\prime}(x) \cup_{1} a_{i}^{\prime \prime}(x)+T_{a}(x, x) .
$$

An easy computation shows that

$$
\delta \theta^{\prime}(x)+\theta^{\prime}(\delta x)=a s q^{n+1}(x)+\sum s q^{n+1+\operatorname{deg} a_{0}^{p}} a_{0}^{p}(x)+e(x),
$$

whenever the left-hand side is defined.

We now apply Lemma 3.4 of [4]. This gives us the existence of an overall defined cochain operation $\theta$ (that is, $\theta \in \mathcal{O}$ ), taking the following values in low dimensions:

for $\operatorname{deg} x \leqq n-2$ :

$$
\theta(x)=0,
$$

for $\operatorname{deg} x=n-1:$

$$
\theta(x)=\Sigma a_{i}^{\prime \prime}(x) a_{i}^{\prime}(\delta x),
$$

for $\operatorname{deg} x=n$ and $\delta x=0$ :

$$
\theta(x)=\Sigma a_{i}^{\prime \prime}(x) a_{i}^{\prime}(x) .
$$


We recall from Section $2((6)$ and (12)) that (in appropriate dimensions) $d(e ; x, y)$ measure the deviation from additivity of the secondary operation $Q u^{s}$, and that $d(\theta ; x, y)$ measure the deviation from additivity of certain tertiary operations. It is therefore of importance to evaluate these cochain operations (in low dimensions).

It is, of course, clear that $d(\psi ; x, y), \psi \in \mathcal{O}$, is not uniquely characterized by the property

$$
\nabla d(\psi ; x, y)+d(\nabla \psi ; x, y)=\psi(x+y)+\psi(x)+\psi(y) .
$$

Anyway, we may define $d(\lambda ; x, y)$ for $\lambda \in F$ in the following way:

$$
\begin{aligned}
d\left(s q^{n+1} ; x, y\right)= & x \cup_{1} y+\delta x \cup_{i+1} y \quad(i=\operatorname{deg} x-n) \\
d(\alpha a ; x, y)= & \alpha d(a ; x, y)+d(\alpha ; a(x), a(y))+ \\
& +d(\alpha ; \nabla d(a ; x, y), a(x)+a(y))+ \\
& +d(\alpha ; \delta d(a ; x, y), d(a ; \delta x, \delta y)), \\
d(\alpha+a ; x, y)= & d(\alpha ; x, y)+d(a ; x, y) .
\end{aligned}
$$

Furthermore, let us define

then

$$
d\left(a ; x_{1}, \ldots, x_{n}\right)=\sum_{i=1}^{n-1} d\left(a ; x_{i}, x_{i+1}+\ldots+x_{n}\right) ;
$$

$$
\delta d\left(a ; x_{1}, \ldots, x_{n}\right)+d\left(a ; \delta x_{1}, \ldots, \delta x_{n}\right)=a\left(\Sigma x_{i}\right)+\Sigma a\left(x_{i}\right) .
$$

It is a consequence of $(6)$ and (7) that $d(e ; x, y)$ vanishes on cochains of dimension less than $n$ and on coycles of dimension $n$, whenever $e$ has excess larger than $n+1$. Note also that $d(a ; x, y)=0$ if $x=0$ or $y=0$.

By means of (6) and (7) we can give the following table for $d(s ; x, y)$, where $s=a s q^{n+1}+\sum s q^{n+1+\operatorname{deg} a_{0}^{p}} a_{0}^{p}+e$ :

$$
\text { for } \operatorname{deg} x=\operatorname{deg} y<n-1 \text { : }
$$

$$
d(s ; x, y)=0,
$$

(8) for $\operatorname{deg} x=\operatorname{deg} y=n-1$ :

$$
\begin{aligned}
d(s ; x, y)=a(\delta x y) & +\Sigma a_{0}^{p}(\delta x) a_{0}^{p}(y)+d(a ; \delta x \delta y, \delta x \delta y)+ \\
& +\Sigma \delta d\left(a_{0}^{p} ; \delta x, \delta y\right)\left(a_{0}^{p}(x)+a_{0}^{p}(y)\right),
\end{aligned}
$$

for $\operatorname{deg} x=\operatorname{deg} y=n$ and $\delta x=\delta y=0$ :

$$
d(s ; x, y)=a(x y)+\Sigma a_{0}^{p}(x) a_{0}^{p}(y)+\Sigma \delta d\left(a_{0}^{p} ; x, y\right)\left(a_{0}^{p}(x)+a_{0}^{p}(y)\right) .
$$

Next, let $\bar{d}(\theta ; x, y)$ be the following partially defined cochain operation: 
for $\operatorname{deg} x=\operatorname{deg} y \leqq n-3$ :

$$
\bar{d}(\theta ; x, y)=0,
$$

for $\operatorname{deg} x=\operatorname{deg} y=n-2$ :

$$
\bar{d}(\theta ; x, y)=\Sigma a_{i}^{\prime \prime}(x) a_{i}^{\prime}(\delta y),
$$

for $\operatorname{deg} x=\operatorname{deg} y=n-1$ :

$$
\begin{aligned}
& \bar{d}(\theta ; x, y)=\sum a_{i}^{\prime \prime}(x) a_{i}^{\prime}(y)+\sum a_{i}^{\prime \prime}(y) \cup_{1} a_{i}^{\prime}(\delta x)+T_{a}(\delta x, y)+ \\
& \quad+\sum d\left(a_{i}^{\prime \prime} ; x, y\right)\left(a_{i}^{\prime}(\delta x)+a_{i}^{\prime}(\delta y)\right)+\Sigma\left(a_{i}^{\prime \prime}(x)+a_{i}^{\prime \prime}(y)\right) d\left(a_{i}^{\prime} ; \delta x, \delta y\right)+ \\
& \quad+\sum d\left(a_{0}^{p} ; \delta x, \delta y\right)\left(a_{0}^{p}(x)+a_{0}^{p}(y)\right)+\operatorname{deg} x d(a ; x \delta y, x \delta y)+ \\
& \quad+\sum d\left(a_{i}^{\prime \prime} ; x, y\right) \delta d\left(a_{i}^{\prime} ; \delta x, \delta y\right),
\end{aligned}
$$

for $\operatorname{deg} x=\operatorname{deg} y=n$ and $\delta x=\delta y=0$ :

$$
\begin{aligned}
& \bar{d}(\theta ; x, y)=T_{a}(x, y)+\sum a_{i}^{\prime \prime}(y) \cup_{1} a_{i}^{\prime}(x)+ \\
& \quad+\sum d\left(a_{i}^{\prime \prime} ; x, y\right)\left(a_{i}^{\prime}(x)+a_{i}^{\prime}(y)\right)+\sum\left(a_{i}^{\prime \prime}(x)+a_{i}^{\prime \prime}(y)\right) d\left(a_{i}^{\prime} ; x, y\right)+ \\
& \quad+\sum d\left(a_{0}^{p} ; x, y\right)\left(a_{0}^{p}(x)+a_{0}^{p}(y)\right)+\sum d\left(a_{i}^{\prime \prime} ; x, y\right) \delta d\left(a_{i}^{\prime} ; x, y\right) .
\end{aligned}
$$

A check gives at once that

$$
\delta \bar{d}(\theta ; x, y)+\bar{d}(\theta ; \delta x, \delta y)=\theta(x)+\theta(y)+\theta(x+y)+d(s ; x, y)
$$

whenever the left-hand side is defined. Here $d(s ; x, y)$ is given in (8) and $\theta(x)$ is given in (5). As before, applying the extension theorem we get an every-where defined cochain operation satisfying (10). It takes the following values in low dimensions:

for $\operatorname{deg} x=\operatorname{deg} y \leqq n-3$ :

$$
d(\theta ; x, y)=0,
$$

for $\operatorname{deg} x=\operatorname{deg} y=n-2$ :

$$
d(\theta ; x, y)=\Sigma a_{i}^{\prime \prime}(x) a_{i}^{\prime}(\delta y),
$$

for $\operatorname{deg} x=\operatorname{deg} y=n-1$ and $\delta x=\delta y=0$ :

$$
d(\theta ; x, y)=\Sigma a_{i}^{\prime \prime}(x) a_{i}^{\prime}(y) .
$$

We shall now turn to the problem of evaluation (in low dimensions) of tertiary cohomology operations. The way we attack this problem is parallel to the case of secondary operations but fairly more complicated and not quite satisfactory.

Let $r: 0=\Sigma \hat{a}_{i} \hat{b}_{i}$ be a relation in $A$, and let 


$$
\begin{aligned}
& \Delta\left(\hat{a}_{i}\right)=\sum \hat{a}_{i, 8}^{\prime} \otimes \hat{a}_{i, s}^{\prime \prime}+\sum \hat{a}_{i, 0}^{p} \otimes \hat{a}_{i, 0}^{p}+\sum \hat{a}_{i, \&}^{\prime \prime} \otimes \hat{a}_{i, 8}^{\prime}, \\
& \Delta\left(\hat{b}_{i}\right)=\sum \hat{b}_{i, t}^{\prime} \otimes \hat{b}_{i, t}^{\prime \prime}+\sum \hat{b}_{i, 0}^{q} \otimes \hat{b}_{i, 0}^{q}+\sum \hat{b}_{i, t}^{\prime \prime} \otimes \hat{b}_{i, t}^{\prime},
\end{aligned}
$$

where $\operatorname{deg} \hat{a}_{i, s}^{\prime}<\operatorname{deg} \hat{a}_{i, s}^{\prime \prime}$ and $\operatorname{deg} \hat{b}_{i, t}^{\prime}<\operatorname{deg} \hat{b}_{i, t}^{\prime \prime}$. Then we have relations

$$
\begin{aligned}
& r_{i}: \quad 0=\hat{b}_{i} S q^{n+1}+\sum S q^{n+1+\operatorname{deg}} \hat{b}_{i, 0}^{q} \hat{b}_{i, 0}^{q}+(\text { terms of excess } \geqq n+2), \\
& r_{i, q}^{\prime}: \quad 0=\hat{a}_{i} S q^{n+1+\operatorname{deg} \hat{b}_{i, 0}^{q}}+\sum S q^{n+1+\frac{1}{2} \operatorname{deg}\left(\hat{a}_{i} \hat{b}_{i}\right)} \hat{a}_{i, 0}^{p}+ \\
& r_{0}: \quad 0=\sum \hat{a}_{i, 0}^{p} \hat{b}_{i, 0}^{q}, \\
& r: 0=\sum \hat{a}_{i} \hat{b}_{i} \text {. } \\
& +(\text { terms of excess } \geqq n+2) \text {, }
\end{aligned}
$$

The cochain operations corresponding to the relations $r, r_{0}, r_{i}$ and $r_{i, q}^{\prime}$ will be denoted by the same symbols. The relations (13) give rise to a relation among relations with an unfactorized relation $\varepsilon$ of excess larger than $n+1$ :

$$
\mathscr{R}: \quad 0=\Sigma a_{i} r_{i}+s q^{n+1+\operatorname{deg} r_{0}} r_{0}+r s q^{n+1}+\sum r_{i, q}^{\prime} b_{i, 0}^{q}+\varepsilon .
$$

(The equation is an equation in the free associative algebra with unit generated by $1, s q^{1}, s q^{2}, \ldots$ )

We recall that to $\mathscr{R}$ in (14) there is associated a Massey product $M$ (see (8) in Section 2). Using the formulas (5), (6) and (7) we get the following expression for $M(x)$ in low dimensions:

$$
\begin{aligned}
& \text { for } \operatorname{deg} x \leqq n-2: \\
& \qquad \begin{aligned}
M(x)= & 0 \\
\text { for } \operatorname{deg} x= & n-1: \\
M(x)= & \sum a_{i}\left(\sum b_{i, t}^{\prime \prime}(x) b_{i, t}^{\prime}(\delta x)\right)+\sum a_{i, s}^{\prime \prime} b_{i, 0}^{q}(x) a_{i, s}^{\prime} b_{i, 0}^{q}(\delta x)+ \\
& +\sum d\left(a_{i} ; \sum b_{i, t}^{\prime \prime}(\delta x) b_{i, t}^{\prime}(\delta x), \sum b_{i, t}^{\prime \prime}(\delta x) b_{i, t}^{\prime}(\delta x)\right)+ \\
& +\sum a_{i, 0}^{p} b_{i, 0}^{q}(\delta x) a_{j, 0}^{u} b_{j, 0}^{v}(x),
\end{aligned}
\end{aligned}
$$

for $\operatorname{deg} x=n$ and $\delta x=0$ :

$$
\begin{aligned}
M(x)= & \sum a_{i}\left(\sum b_{i, t}^{\prime \prime}(x) b_{i, t}^{\prime}(x)\right)+\sum a_{i, s}^{\prime \prime} b_{i, 0}^{q}(x) a_{i, s}^{\prime} b_{i, 0}^{q}(x)+ \\
& +\sum a_{i, 0}^{p} b_{i, 0}^{q}(x) a_{j, 0}^{u} b_{j, 0}^{v}(x) .
\end{aligned}
$$

The last summation in both of the cases $\operatorname{deg} x=n-1$ and $\operatorname{deg} x=n$ is over all triples $(i, p, q)$ and $(j, u, v)$, where $(i, p, q)<(j, u, v)$ for some ordering of triples of indices $(i, p, q)$. We make this a convention such that

$$
\sum a_{i, 0}^{p} b_{i, 0}^{q}(x) a_{j, 0}^{u} b_{j, 0}^{v}(y)
$$

in the rest of this paper will mean summation over all $(i, p, q)$ and $(j, u, v)$ with $(i, p, q)<(j, v, u)$. 
As a consequence of (15) we see that the cohomology operation $\hat{M}$ associated to $M$ has excess larger than $n-1$. Hence there is a formula

$$
\hat{M}=\Sigma S q^{I} S q^{n+\operatorname{deg} J} S q^{J}+\hat{e},
$$

where the terms $S q^{I} S q^{n+\operatorname{deg} J} S q^{J}$ are admissible of excess $n$ and $\hat{e}$ is admissible of excess larger than $n$. Note in particular that

$$
s q^{I} s q^{n+\operatorname{deg} J} s q^{J}(x)=s q^{I_{0}} s q^{J}(x) s q^{I_{0}} s q^{J}(x)
$$

if $x$ is an $n$-dimensional cocycle, and that

$$
s q^{I} s q^{n+\operatorname{deg} J} s q^{J}(x)=s q^{I_{0}} s q^{J}(x) s q^{I_{0}} s q^{J}(\delta x)
$$

if $x$ is a $(n-1)$-dimensional cochain.

The aim of the rest of this section is, in low dimensions, to give an explicit expression for a cochain operation $\chi$, satisfying

$$
\nabla \chi=M+\Sigma s q^{I} s q^{n+\operatorname{deg} J} s q^{J}+e .
$$

Before we can state the result, we have to make some preparations. Let us define $\Sigma e_{\nu}^{\prime \prime} f_{v}^{\prime \prime} \otimes e_{v}^{\prime} f_{v}^{\prime}$ by

$$
\begin{aligned}
\sum e_{v}^{\prime \prime} f_{\nu}^{\prime \prime} \otimes e_{v}^{\prime} f_{,}^{\prime}= & \sum a_{i} b_{i} \otimes 1+\sum a_{i, s}^{\prime \prime} b_{i, t}^{\prime \prime} \otimes a_{i, s}^{\prime} b_{i, t}^{\prime}+ \\
& +\uparrow \sum a_{i, s}^{\prime \prime} b_{i, t}^{\prime} \otimes a_{i, s}^{\prime} b_{i, s}^{\prime \prime}+\uparrow \sum a_{i, s}^{\prime} b_{i, t}^{\prime \prime} \otimes a_{i, s}^{\prime \prime} b_{i, t}^{\prime}+ \\
& +\sum a_{i, s}^{\prime \prime} b_{i, 0} \otimes a_{i, s}^{\prime} b_{i, 0}+\sum a_{i, 0} b_{i, t}^{\prime \prime} \otimes a_{i, 0} b_{i, t}^{\prime} .
\end{aligned}
$$

The equality is considered as an equality in $F \otimes F$ (see Section 2 (1) for definition of $F^{\prime}$ ). The Cartan formula (in $F$ ) of $\sum a_{i} b_{i}$ then takes the following form:

$$
\begin{aligned}
\Delta \sum a_{i} b_{i}= & 1 \otimes \sum a_{i} b_{i}+\sum e_{v}^{\prime} f_{v}^{\prime} \otimes e_{\nu}^{\prime \prime} f_{\nu}^{\prime \prime}+\left\{\sum a_{i, s}^{\prime} b_{i, t}^{\prime \prime} \otimes a_{i, s}^{\prime \prime} b_{i, t}^{\prime}+\right. \\
& +\uparrow \sum a_{i, s}^{\prime \prime} b_{i, t}^{\prime} \otimes a_{i, s}^{\prime} b_{i, t}^{\prime \prime}+\sum a_{i, 0}^{p} b_{i, 0}^{q} \otimes a_{i, 0}^{p} b_{i, 0}^{q}+ \\
& +\sum a_{i} b_{i} \otimes 1+\sum e_{v}^{\prime \prime} f_{\nu}^{\prime \prime} \otimes e_{\nu}^{\prime} f_{\nu}^{\prime} .
\end{aligned}
$$

Here $\uparrow \Sigma(-\otimes-)(\downarrow \Sigma-\otimes-, \uparrow \Sigma-\otimes-)$ is a short notation for that part of the sum, where the degree of the left-hand term is larger (less than, equal to) the degree of the right-hand term.

The admissible monomials constitute an additive basis for $A$. Hence there exist admissible monomials

such that

$$
\begin{array}{ll}
m_{k}^{\prime}, & k \in \cup M_{v}^{\prime}, \\
m_{h}^{\prime \prime}, & h \in \cup M_{v}^{\prime \prime},
\end{array}
$$

$$
\begin{array}{cl}
s_{v}^{\prime}=e_{v}^{\prime} f_{v}^{\prime}+\sum m_{k}^{\prime}, & k \in M_{v}^{\prime}, \\
s_{v}^{\prime \prime}=e_{v}^{\prime \prime} f_{v}^{\prime \prime}+\sum m_{h}^{\prime \prime}, & h \in M_{v}^{\prime \prime},
\end{array}
$$


are relations. We have the following identities in $F \otimes F$

$$
\begin{aligned}
\Sigma e_{v}^{\prime \prime} f_{\nu}^{\prime \prime} \otimes e_{v}^{\prime} f_{\nu}^{\prime} & =\Sigma s_{v}^{\prime \prime} \otimes e_{v}^{\prime} f_{v}^{\prime}+\Sigma_{v}\left(\Sigma m_{h}^{\prime \prime}\right) \otimes s_{v}^{\prime}, \\
\Sigma_{v}\left(\Sigma m_{h}^{\prime \prime}\right) \otimes\left(\Sigma m_{k}^{\prime}\right) & =0 .
\end{aligned}
$$

In (15) we gave a table for the Massey product $M(x)$. By a short computation one sees that $M(x)$ is cohomologous to

$$
\left\{\sum a_{i, s}^{\prime} b_{i, t}^{\prime \prime}(x) a_{i, s}^{\prime \prime} b_{i, t}^{\prime}(x)+\sum a_{i, 0}^{p} b_{i, 0}^{q}(x) a_{j, 0}^{u} b_{j, 0}^{v}(x),\right.
$$

when $x$ is an $n$-dimensional cocycle. Thus by (16a) the following expression is cohomologous to zero:

$$
\begin{aligned}
\uparrow \sum a_{i, s}^{\prime} b_{i, t}^{\prime \prime}(x) a_{i, s}^{\prime \prime} b_{i, t}^{\prime}(x) & +\sum a_{i, 0}^{p} b_{i, 0}^{q}(x) a_{j, 0}^{u} b_{j, 0}^{v}(x)+ \\
& +\sum s q^{I_{0}} s q^{J}(x) s q^{I_{0}} s q^{J}(x) .
\end{aligned}
$$

Here $I_{0}$ denotes the "half of $I$ ". We introduce a short notation for (22):

Now, choose admissible monomials

$$
\begin{aligned}
\Sigma g_{\lambda}^{\prime} h_{\lambda}^{\prime} \otimes g_{\lambda}^{\prime \prime} h_{\lambda}^{\prime \prime}=\uparrow \Sigma a_{i, s}^{\prime} b_{i, t}^{\prime \prime} \otimes a_{i, s}^{\prime \prime} b_{i, t}^{\prime} & +\sum a_{i, 0}^{p} b_{i, 0}^{q} \otimes a_{j, 0}^{u} b_{j, 0}^{v}+ \\
& +\sum s q^{I_{0}} s q^{J} \otimes s q^{I_{0}} s q^{J}
\end{aligned}
$$

such that

$$
\begin{aligned}
& \bar{m}_{\omega}^{\prime}, \quad \omega \in \bigcup \bar{M}_{\lambda}^{\prime}, \\
& \bar{m}_{\pi}^{\prime \prime}, \quad \pi \in \bigcup \bar{M}_{\lambda}^{\prime \prime},
\end{aligned}
$$

and

$$
t_{\lambda}^{\prime}=g_{\lambda}^{\prime} h_{\lambda}^{\prime}+\Sigma \bar{m}_{\omega}^{\prime}, \quad \omega \in \bar{M}^{\prime},
$$

$$
t_{\lambda}^{\prime \prime}=g_{\lambda}^{\prime \prime} h_{\lambda}^{\prime \prime}+\Sigma \bar{m}_{\pi}^{\prime \prime}, \quad \pi \in \bar{M}^{\prime \prime},
$$

are relations. We have the following equation in $F \otimes F$ :

$$
\Sigma g_{\lambda}^{\prime} h_{\lambda}^{\prime} \otimes g_{\lambda}^{\prime \prime} h_{\lambda}^{\prime \prime}=\Sigma t_{\lambda}^{\prime} \otimes g_{\lambda}^{\prime \prime} h_{\lambda}^{\prime \prime}+\Sigma_{\lambda}\left(\Sigma \bar{m}_{\omega}^{\prime}\right) \otimes t_{\lambda}^{\prime \prime}+\Sigma_{\lambda}\left(\Sigma \bar{m}_{\omega}^{\prime}\right) \otimes\left(\Sigma \bar{m}_{\pi}^{\prime \prime}\right) \text {. }
$$

Let $\hat{z}^{n}$ be the fundamental cohomology class in $H^{n}\left(K\left(Z_{2}, n\right)\right)$. We have

$$
\Sigma_{\lambda}\left(\sum \hat{\bar{m}}_{\omega}^{\prime}\left(\hat{z}^{n}\right)\right)\left(\sum \hat{\bar{m}}_{\pi}^{\prime \prime}\left(\hat{z}^{n}\right)\right)=0
$$

since $\sum g_{\lambda}^{\prime} h_{\lambda}^{\prime}\left(z^{n}\right) g_{\lambda}^{\prime \prime} h_{\lambda}^{\prime \prime}\left(z^{n}\right)$ by $(22)$ is null-cohomologous. Hence there exist admissible monomials $n_{\mu}^{\prime}$ and $n_{\mu}^{\prime \prime}$ such that (in $F \otimes F$ )

$$
\Sigma_{\lambda}\left(\Sigma \bar{m}_{\omega}^{\prime}\right) \otimes\left(\Sigma \bar{m}_{\pi}^{\prime \prime}\right)=\Sigma n_{\mu}^{\prime} \otimes n_{\pi}^{\prime \prime}+\Sigma n_{\mu}^{\prime \prime} \otimes n_{\mu}^{\prime}+\Sigma \varrho^{\prime} \otimes \varrho^{\prime \prime},
$$

where each summand in the last term has the property that either $\varrho^{\prime}$ or $\varrho^{\prime \prime}$ has excess larger than $n$.

We are now ready to state the main result of this section.

First, let us choose cochain operations $R, S_{v}^{\prime}, S_{v}^{\prime \prime}, T_{\lambda}^{\prime}$ and $T_{\lambda}^{\prime \prime}$ in $\mathcal{O}$ such that 


$$
\nabla R=r, \quad \nabla S_{v}^{\prime}=s_{v}^{\prime}, \quad \nabla S_{v}^{\prime \prime}=s_{v}^{\prime \prime}, \quad \nabla T_{\lambda}^{\prime}=t_{\lambda}^{\prime}, \quad \nabla T_{\lambda}^{\prime \prime}=t_{\lambda}^{\prime \prime} .
$$

Then define $\chi^{\prime}$ as follows:

for $\operatorname{deg} x \leqq n-3$ :

$$
\chi^{\prime}(x)=0,
$$

for $\operatorname{deg} x=n-2$ :

$$
\begin{aligned}
\chi^{\prime}(x)=\downarrow \sum a_{i, s}^{\prime} b_{i, t}^{\prime \prime}(x) a_{i, s}^{\prime \prime} b_{i, t}^{\prime}(\delta x) & +\sum a_{i, 0}^{p} b_{i, 0}^{q}(x) a_{j, 0}^{u} b_{j, 0}^{v}(\delta x)+ \\
& +\sum n_{\mu}^{\prime \prime}(x) n_{\mu}^{\prime}(\delta x),
\end{aligned}
$$

for $\operatorname{deg} x=n-1$ :

$$
\begin{aligned}
\chi^{\prime}(x)= & \downarrow \sum a_{i, s}^{\prime} b_{i, t}^{\prime \prime}(x) a_{i, s}^{\prime \prime} b_{i, t}^{\prime}(x)+\sum a_{i, 0}^{p} b_{i, 0}^{q}(x) a_{j, 0}^{u} b_{j, 0}^{v}(x)+ \\
& +\sum n_{\mu}^{\prime \prime}(x) n_{\mu}^{\prime}(x)+R(x) \delta x+\sum S_{\nu}^{\prime \prime}(x) e_{\nu}^{\prime} f_{\nu}^{\prime}(\delta x)+ \\
& +\sum{ }_{\nu}\left(\sum m_{h}^{\prime \prime}(x)\right) S_{\nu}^{\prime}(\delta x)+\sum T_{\lambda}^{\prime}(x) g_{\lambda}^{\prime \prime} h_{\lambda}^{\prime \prime}(\delta x)+\sum_{\lambda}\left(\sum \bar{m}_{\omega}^{\prime}(x)\right) T_{\lambda}^{\prime \prime}(\delta x)+ \\
& +\downarrow \sum a_{i, s}^{\prime} b_{i, t}^{\prime \prime}(\delta x) \cup_{1} a_{i, s}^{\prime \prime} b_{i, t}^{\prime}(x)+\sum n_{\mu}^{\prime}(x) \cup_{1} n_{\mu}^{\prime \prime}(\delta x)+ \\
& +\sum T_{a_{i}}\left(b_{i, t}^{\prime \prime}(x), b_{i, t}^{\prime}(\delta x)\right)+\sum d\left(a_{i} ; \ldots, b_{i, t}^{\prime \prime}(x) b_{i, t}^{\prime}(\delta x), \ldots\right),
\end{aligned}
$$

for $\operatorname{deg} x=n$ and $\delta x=0$ :

$$
\begin{aligned}
\chi^{\prime}(x)= & R(x) x+\sum S_{\nu}^{\prime \prime}(x) e_{v}^{\prime} f_{\nu}^{\prime}(x)+\sum_{v}\left(\sum m_{h}^{\prime \prime}(x)\right) S_{v}^{\prime}(x)+ \\
& +\sum T_{\lambda}^{\prime}(x) g_{\lambda}^{\prime \prime} h_{\lambda}^{\prime \prime}(x)+\sum_{\lambda}\left(\sum \bar{m}_{\omega}^{\prime}(x)\right) T_{\lambda}^{\prime \prime}(x)+ \\
& +\downarrow \sum a_{i, s}^{\prime} b_{i, t}^{\prime \prime}(x) \cup_{1} a_{i, s}^{\prime \prime} b_{i, t}^{\prime}(x)+\sum n_{\mu}^{\prime}(x) \cup_{1} n_{\mu}^{\prime \prime}(x)+ \\
& +\sum T_{a_{i}}\left(b_{i, t}^{\prime \prime}(x), b_{i, t}^{\prime}(x)\right)+\sum d\left(a_{i} ; \ldots, b_{i, t}^{\prime \prime}(x) b_{i, t}^{\prime}(x), \ldots\right)+ \\
& +\sum d\left(a_{i} ; \sum b_{i, t}^{\prime \prime}(x) b_{i, t}^{\prime}(x), \sum b_{i, t}^{\prime \prime}(x) b_{i, t}^{\prime}(x)\right) .
\end{aligned}
$$

An easy but rather lengthy computation shows that

$$
\delta \chi^{\prime}(x)+\chi^{\prime}(\delta x)=M(x)+s q^{I} s q^{\operatorname{deg} J+n} s q^{J}(x)+e(x)
$$

whenever this makes sense. By Lemma 3.4 of [4] there exists a cochain operation $\chi$ such that:

for $\operatorname{deg} x \leqq n-1$ and $\delta x=0$ :

$$
\chi(x)=\chi^{\prime}(x),
$$

for $\operatorname{deg} x=n$ and $\delta x=0$ :

$$
\chi(x)=\chi^{\prime}(x)+\Sigma s q^{I_{1}}(x) s q^{I_{2}}(x) \cdot \ldots \cdot s q^{I_{r}}(x), \quad r \geqq 2 .
$$

\section{Theorems.}

All references in this section are references to Section 3.

Let $s$ be the relation (1) and $\mathscr{R}$ the relation among relations (14). Then we have 
Theorem 1. There exists a secondary operation associated with the relation $s$, taking the following values in low dimensions:

$$
\begin{array}{r}
\text { for } \operatorname{deg} \hat{x} \leqq n-1: \\
Q u^{8}(\widehat{x})=0,
\end{array}
$$

for $\operatorname{deg} \hat{x}=n$ :

$$
Q u^{s}(\hat{x})=\Sigma \hat{a}_{i}^{\prime \prime}(\hat{x}) \hat{a}_{i}^{\prime}(\hat{x}) \quad\left(\frac{1}{2} \text { Cartan formula }\right) .
$$

Proof. Apply (5).

TheOREM 2. There is a tertiary operation associated with the relation among relations $\mathscr{R}$, taking the following values in low dimensions:

a) for $\operatorname{deg} \hat{x} \leqq n-2$ :

$$
Q u^{\mathscr{R}}(\hat{x})=0,
$$

b) for $\operatorname{deg} \hat{x}=n-1$ :

$$
\begin{aligned}
Q u^{\mathscr{R}}(\hat{x})=\downarrow \sum \hat{a}_{i, s}^{\prime} \hat{b}_{i, t}^{\prime \prime}(\hat{x}) \hat{a}_{i, s}^{\prime \prime} \hat{b}_{i, t}^{\prime}(\hat{x}) & +\sum \hat{a}_{i, 0}^{p} \hat{b}_{i, 0}^{q}(\hat{x}) \hat{a}_{j, 0}^{u} \hat{b}_{j, 0}^{v}(\hat{x})+ \\
& +\sum \hat{n}_{\mu}^{\prime \prime}(\hat{x}) \hat{n}_{\mu}^{\prime}(\hat{x}),
\end{aligned}
$$

c) for $\operatorname{deg} \hat{x}=n$ and $\hat{x}$ annihilated by all primary operations of degree less than $\operatorname{deg} \mathscr{R}-n$ :

$$
Q u^{\mathscr{R}}(\hat{x})=Q u^{r}(\hat{x}) \hat{x} \quad\left(\frac{1}{2} \text { Cartan formula }\right) .
$$

Remark. The symbol $\downarrow \Sigma$ is explained in (19). The term

$$
\sum a_{i, 0}^{p} t_{i, 0}^{q} \otimes a_{j, 0}^{u} l_{j, 0}^{v}
$$

is defined in (15), and the term $\Sigma n_{\mu}^{\prime} \otimes n_{\mu}^{\prime \prime}$ in (24). Note that these two terms are zero if $\operatorname{deg}\left(\hat{a}_{i} \hat{b}_{i}\right)$ is odd.

Proof of Theorem 2. Part a) and part $b$ ) are immediate consequences of (25). In order to prove c) we introduce the notation $\delta^{-1} b$ for a cochain with $\delta\left(\delta^{-1} b\right)=b$. This is a convenient abuse of notation; handled with care it will not give rise to confusions. From (11) of Section 2 one sees that $Q u^{\mathscr{R}}(\hat{x})(\operatorname{deg} \hat{x}=n)$ is represented by the cocycle:

$$
\begin{aligned}
q u^{\mathscr{R}}(x)= & \chi(x)+\sum a_{i} \delta^{-1} q u^{r_{i}}(x)+\sum R_{i, q}^{\prime} \delta^{-1} l_{i, 0}^{q}(x)+ \\
& +\Sigma^{*} s q^{I} s q^{\operatorname{deg} J+n} \delta^{-1} s q^{J}(x)+s q^{I} \delta^{-1} s q^{n}(x)+ \\
& +d\left(s q^{n+1+\operatorname{deg} r_{0}} ; \ldots, a_{i, 0}^{p} \delta^{-1} b_{i, 0}^{q}(x), \ldots, q u^{r_{0}}\left(x,\left\{\delta^{-1} l_{i, 0}^{q}(x)\right\}\right)\right),
\end{aligned}
$$

where $\nabla R_{i, q}^{\prime}=r_{i, q}^{\prime}$ and $\Sigma^{*} s q^{I} s q^{\operatorname{deg} J+n} s q^{J}$ is the part of the sum $\sum s q^{I} s q^{\operatorname{deg} J+n} s q^{J}$, where $s q^{J} \neq 1$. By (5) we have

Math. Scand. $20-9$ 


$$
\begin{aligned}
q u^{\mathscr{T}}(x) \sim \chi(x) & +\sum a_{i}\left(\sum \delta^{-1} b_{i, t}^{\prime \prime}(x) b_{i, t}^{\prime}(x)\right)+ \\
& +\sum a_{i, s}^{\prime \prime} \delta^{-1} b_{i, 0}^{q}(x) a_{i, s}^{\prime} b_{i, 0}^{q}(x)+\sum a_{i, 0}^{p} b_{i, 0}^{q}(x) a_{j, 0}^{u} \delta^{-1} b_{j, 0}^{v}(x)+ \\
& +\Sigma^{*} s q^{I} s q^{n+\operatorname{deg} J} \delta^{-1} s q^{J}(x)+s q^{I} \delta^{-1}\left(s q^{n} x\right) .
\end{aligned}
$$

By (18), (19) and (25)

$$
\begin{aligned}
q u^{\mathscr{R}}(x) \sim & R(x) x+\left(\sum a_{i} \delta^{-1} b_{i}(x)\right) x+\uparrow \sum a_{i, s}^{\prime} \delta^{-1} b_{i, t}^{\prime \prime}(x) a_{i, s}^{\prime \prime} b_{i, t}^{\prime}(x)+ \\
& +\sum a_{i, 0}^{p} b_{i, 0}^{q}(x) a_{j, 0}^{u} \delta^{-1} b_{j, 0}^{v}(x)+\sum n_{\mu}^{\prime}(x) \delta^{-1} n_{\mu}^{\prime \prime}(x)+ \\
& +\sum \delta^{-1} n_{\mu}^{\prime \prime}(x) n_{\mu}^{\prime}(x)+\sum t_{\lambda}^{\prime}(x) \delta^{-1} g_{\lambda}^{\prime \prime} h_{\lambda}^{\prime \prime}(x)+\sum_{\lambda}\left(\sum \delta^{-1} \bar{m}_{\omega}^{\prime}(x)\right) t_{\lambda}^{\prime \prime}(x)+ \\
& +\Sigma^{*} s q^{I} s q^{n+\operatorname{deg} J} \delta^{-1} s q^{J}(x)+s q^{I}\left(\delta^{-1} s q^{n} x\right) .
\end{aligned}
$$

Hence by (16a), (16b), (22), (23) and (24)

$$
q u^{\mathscr{R}}(x) \sim R(x) x+\left(\sum a_{i} \delta^{-1} b_{i}(x)\right) x=q u^{r}\left(x,\left\{\delta^{-1} b_{i}(x)\right\}\right) x .
$$

This completes the proof.

\section{BIBLIOGRAPHY}

1. J. F. Adams, On the non-existence of elements of Hopf invariant one, Ann. of Math. 72 (1960), 20-104.

2. A. Kock and L. Kristensen, $A$ secondary product structure in cohomology theory, Math. Scand. 17 (1965), 57-82.

3. A. Kock, L. Kristensen, and I. Madsen, Cochainfunctors for general cohomology theories I-II, Math. Scand. 20 (1967), 131-150, 151-176.

4. L. Kristensen, On secondary cohomology operations, Math. Scand. 12 (1963), 57-82.

5. L. Kristensen, On a Cartan formula for secondary cohomology operations, Math. Scand. 16 (1965), 97-115.

6. M. E. Mahowald, Some Whitehead products in $S^{n}$, Topology 4 (1965), 17-26.

7. C. R. F. Maunder, Cohomology operations of the Nth kind, Proc. London Math. Soc. (3) 13 (1963), 125-154. 